\title{
An Algorithm for Learning from Hints
}

\author{
Y. S. Abu-Mostafa \\ California Institute of Technology, Pasadena, CA 91125, USA
}

\begin{abstract}
To take advantage of prior knowledge (hints) about the function we want to learn, we introduce a method that generalizes learning from examples to learning from hints. A canonical representation of hints is defined and illustrated. All hints are represented to the learning process by examples, and examples of the function are treated on equal footing with the rest of the hints. During learning, examples from different hints are selected for processing according to a given schedule. We present two types of schedules; fixed schedules that specify the relative emphasis of each hint, and adaptive schedules that are based on how well each hint has been learned so far. Our learning method is compatible with any descent technique.
\end{abstract}

\section{Introduction}

Consider the situation where we want to learn an unknown function $f$. Typically, $f$ is represented to us by a set of input-output examples. Hints describe the situation where, in addition to the set of examples of $f$, we have prior knowledge of certain facts about the function. We wish to use this side information to our advantage. However, hints come in different shapes, and the main difficulty of using them is the lack of a systematic way of incorporating heterogeneous pieces of information into a manageable learning process. If what we know about $f$ is that it is scale-invariant, monotonic over part of its domain, and represented by a given set of examples, we still have to integrate this information before we can learn the function. This paper concerns itself with the development of a systematic method that integrates different types of hints in the same learning process.

How to take advantage of a given hint can be an art just like how to choose a learning model. In the case of invariance hints for instance, preprocessing of the input can achieve the invariance through normalization, or the model itself can be explicitly structured to satisfy the invariance. Whenever such a method of direct implementation is feasible, the full benefit of the hint is automatically realized. This paper does not offer a superior alternative to direct implementation. However, when direct implementation is not an option, we prescribe a systematic method for incorporating practically any hint in any descent method for learning. The goal is to automate the use of hints in learning to a degree where we can effectively use a large number of simple hints that may be available in a practical situation.

We start by introducing the basic nomenclature and notation. The environment $X$ is the set on which the unknown function $f$ is defined. The points in the environment are distributed according to some probability distribution $P . f$ takes on values from some set $Y$

$$
f: X \rightarrow Y
$$

Often, $Y$ is just $\{0,1\}$ or the interval $[0,1]$. The learning process takes pieces of information about (the otherwise unknown) $f$ as input and produces a hypothesis $g$

$$
g: X \rightarrow Y
$$

that attempts to approximate $f$. The degree to which a hypothesis $g$ is considered an approximation of $f$ is measured by a distance or 'error'

$$
E(g, f)
$$

The error $E$ is based on the disagreement between $g$ and $f$ as seen through the eyes of the probability distribution $P$.

Two popular forms of the error measure are

$$
E=\operatorname{Pr}[g(x) \neq f(x)]
$$

and

$$
E=\mathcal{E}\left[(g(x)-f(x))^{2}\right]
$$


where $\operatorname{Pr}[$.$] denotes the probability of an event, and$ $\mathcal{E}[$.$] denotes the expected value of a random vari-$ able. The underlying probability distribution is $P$. $E$ will always be a non-negative quantity, and we will take $E(g, f)=0$ to mean that $g$ and $f$ are identical for all intents and purposes. We will also assume that when the set of hypotheses is parameterized by real-valued parameters (e.g., the weights in the case of a neural network), $E$ will be wellbehaved as a function of the parameters (in order to allow for derivative-based descent methods). We make the same assumptions about the error measures that will be introduced in section 2 for the hints.

In the learning from examples paradigm, a number of points $x_{1}, \cdots, x_{N}$ are picked from $X$ (usually independently according to the probability distribution $P$ ) and the values of $f$ on these points are provided. Thus, the input to the learning process is the set of examples

$$
\left(x_{1}, f\left(x_{1}\right)\right), \cdots,\left(x_{N}, f\left(x_{N}\right)\right)
$$

and these examples are used to guide the search for a good hypothesis. In this paper, we will consider the set of examples of $f$ as only one of the available hints and denote it by $H_{0}$. The other hints $H_{1}, \cdots, H_{M}$ will be additional known facts about $f$, such as invariance properties for instance.

The paper is organized as follows. Section 2 develops a canonical method for representing different hints. This is the first step in dealing with any hint that we encounter in a practical situation. Section 3 lays the foundations for learning from hints in general, and section 4 presents specific implementations. We discuss the overall picture in the conclusion.

\section{Representation of Hints}

We have so far described what a hint is in very general terms such as 'a known property of $f$ ' or 'a fact about $f$ '. Indeed, all that is needed to qualify as a hint for our purposes is to have a litmus test that $f$ passes and that can be applied to the set of hypotheses. In other words, a hint $H_{m}$ is formally a subset of the hypotheses, namely those satisfying the hint.

This definition of $H_{m}$ can be extended to a definition of 'approximation of $H_{m}$ ' in several ways. For instance, $g$ can be considered to approximate $H_{m}$ within $\epsilon$ if there is a function $h$ that strictly satisfies $H_{m}$ for which $E(g, h) \leq \epsilon$. In the context of learning, it is essential to have a notion of approximation since exact learning is seldom achievable. Our definitions for approximating different hints will be part of the scheme for representing those hints.

The first step in representing $H_{m}$ is to choose a way of generating 'examples' of the hint. For illustration, suppose that $H_{m}$ asserts that

$$
f:[-1,+1] \rightarrow[-1,+1]
$$

is an odd function. An example of $H_{m}$ would have the form

$$
f(-x)=-f(x)
$$

for a particular $x \in[-1,+1]$. To generate $N$ examples of this hint, we generate $x_{1}, \cdots, x_{N}$ and assert for each $x_{n}$ that $f\left(-x_{n}\right)=-f\left(x_{n}\right)$. Suppose that we are in the middle of a learning process, and that the current hypothesis is $g$ when the example $f(-x)=-f(x)$ is presented. We wish to measure how. much $g$ disagrees with this example. This leads to the second component of the representation, the error measure $e_{m}$. For the oddness hint, $e_{m}$ can be defined as

$$
e_{m}=(g(x)+g(-x))^{2}
$$

so that $e_{m}=0$ reflects total agreement with the example (i.e., $g(-x)=-g(x)$ ). The form of the examples of $H_{m}$ as well as the choice of the error measure $e_{m}$ are not unique.

Once the disagreement between $g$ and an example of $H_{m}$ has been quantified through $e_{m}$, the disagreement between $g$ and $H_{m}$ as a whole is automatically quantified through $E_{m}$, where

$$
E_{m}=\mathcal{E}\left(e_{m}\right)
$$

The expected value is taken w.r.t. the probability rule for picking the examples. This rule is also not unique. Therefore, $E_{m}$ will depend on our choices in all three components of the representation; the form of examples, the probability distribution for picking the examples, and the error measure $e_{m}$. In what follows, we will construct representations for different types of hints.

Perhaps the most common type of hint is the invariance hint. This hint asserts that $f(x)=$ $f\left(x^{\prime}\right)$ for certain pairs $x, x^{\prime}$. For instance, " $f$ is shift-invariant" is formalized by the pairs $x, x^{\prime}$ that are shifted versions of each other. To represent the invariance hint, an invariant pair $\left(x, x^{\prime}\right)$ is picked as an example. The error associated with this example is

$$
e_{m}=\left(g(x)-g\left(x^{\prime}\right)\right)^{2}
$$


A plausible probability rule for generating $\left(x, x^{\prime}\right)$ is to pick $x$ and $x^{\prime}$ according to the original probability distribution $P$ conditioned on $x, x^{\prime}$ being an invariant pair.

Another related type of hint is the monotonicity hint (or inequality hint). The hint asserts for certain pairs $x, x^{\prime}$ that $f(x) \leq f\left(x^{\prime}\right)$. For instance, " $f$ is monotonically nondecreasing in $x$ " is formalized by all pairs $x, x^{\prime}$ such that $x \leq x^{\prime}$. To represent a monotonicity hint, an example $\left(x, x^{\prime}\right)$ is picked, and the error associated with this example is

$$
e_{m}= \begin{cases}\left(g(x)-g\left(x^{\prime}\right)\right)^{2} & \text { if } g(x)>g\left(x^{\prime}\right) \\ 0 & \text { if } g(x) \leq g\left(x^{\prime}\right)\end{cases}
$$

The third type of hint we discuss here is the approximation hint. The hint asserts for certain points $x \in X$ that $f(x) \in\left[a_{x}, b_{x}\right]$. In other words, the value of $f$ at $x$ is known only approximately. The error associated with an example $x$ of the approximation hint is

$$
e_{m}= \begin{cases}\left(g(x)-a_{x}\right)^{2} & \text { if } g(x)<a_{x} \\ \left(g(x)-b_{x}\right)^{2} & \text { if } g(x)>b_{x} \\ 0 & \text { if } g(x) \in\left[a_{x}, b_{x}\right]\end{cases}
$$

Another type of hints arises when the learning model allows non-binary values for $g$ where $f$ itself is known to be binary. This gives rise to the binary hint (or Boolean hint). Let $\hat{X} \subseteq X$ be the set where $f$ is known to be binary (for Boolean functions, $\hat{X}$ is the set of binary input vectors). The binary hint is represented by examples of the form $x$, where $x \in \hat{X}$. The error function associated with an example $x$ (assuming $0 / 1$ binary convention, and assuming $g(x) \in[0,1])$ is

$$
e_{m}=g(x)(1-g(x))
$$

This choice of $e_{m}$ forces it to be zero when $g(x)$ is either 0 or 1 , while it would be positive if $g(x)$ is between 0 and 1. A natural probability rule for generating the examples is to pick $x$ according to the original probability distribution $P$ conditioned on $x \in \hat{X}$.

It is worth noting that the set of examples of $f$ can be formally treated as a hint, too. Given $\left(x_{1}, f\left(x_{1}\right)\right), \cdots,\left(x_{N}, f\left(x_{N}\right)\right)$, the examples hint asserts that these are the correct values of $f$ at these particular points $x_{n}$. Now, to generate an 'example' of this hint, we independently pick a number $n$ from 1 to $N$ and use the corresponding $\left(x_{n}, f\left(x_{n}\right)\right)$. The error associated with this example is $e_{0}$ (we fix the convention that $m=0$ for the examples hint)

$$
e_{0}=\left(g\left(x_{n}\right)-f\left(x_{n}\right)\right)^{2}
$$

Assuming that the probability rule for picking $n$ is uniform over $\{1, \cdots, N\}$,

$$
E_{0}=\mathcal{E}\left(e_{0}\right)=\frac{1}{N} \sum_{n=1}^{N}\left(g\left(x_{n}\right)-f\left(x_{n}\right)\right)^{2}
$$

In this case, $E_{0}$ is also the best estimator of $E=$ $\mathcal{E}\left[(g(x)-f(x))^{2}\right]$ given $x_{1}, \cdots, x_{N}$ that are independently picked according to the original probability distribution $P$. This way of looking at the examples of $f$ justifies their treatment exactly as one of the hints, and underlines the distinction between $E$ and $E_{0}$.

In a practical situation, we try to infer as many hints about $f$ as the situation will allow. Next, we represent each hint according to the guidelines discussed in this section. This leads to a list $H_{0}, H_{1}, \cdots, H_{M}$ of hints that are ready to produce examples upon the request of the learning algorithm. We now address how the algorithm should pick and choose between these examples as it moves along.

\section{Learning Schedules}

If the learning algorithm had complete information about $f$, it would search for a hypothesis $g$ for which $E(g, f)=0$. However, $f$ being unknown means that the point $E=0$ cannot be directly identified. The most any learning algorithm can do given the hints $H_{0}, H_{1}, \cdots, H_{M}$ is to reach a hypothesis $g$ for which all the error measures $E_{0}, E_{1}, \cdots, E_{M}$ are zeros. Indeed, we have required that $E=0$ implies that $E_{m}=0$ for all $m$.

If that point is reached, regardless of how it is reached, the job is done. However, it is seldom the case that we can reach the zero-error point because either (1) it does not exist (i.e., no hypothesis can satisfy all the hints sirnultaneously, which implies that no hypothesis can replicate $f$ exactly), or (2) it is difficult to reach (i.e., the computing resources do not allow us to exhaustively search the space of hypotheses looking for that point). In either case, we will have to settle for a point where the $E_{m}$ 's are 'as small as possible'.

How small should each $E_{m}$ be? A balance has to be struck, otherwise some $E_{m}$ 's may become very small at the expense of the others. This situation would mean that some hints are over-learned while the others are under-learned. We will discuss learning schedules that use different criteria for balancing between the hints. The schedules are used by the learning algorithm to simultaneously minimize the $E_{m}$ 's. 
The implementation of a given schedule goes as follows: (1) The algorithm decides which hint (which $m$ for $m=0,1, \cdots, M$ ) to work on next, according to some criterion; (2) The algorithm then requests a batch of examples of this hint; (3) It performs its descent on this batch; and (4) When it is done, it goes back to step (1). We make a distinction between fixed schedules, where the criterion for selecting the hint can be 'evaluated' ahead of time (albeit time-invariant or time-varying, deterministic or stochastic), and adaptive schedules, where the criterion depends on what happens as the algorithm runs. Here are some fixed and adaptive schedules:

Simple Rotation: This is the simplest possible schedule that tries to balance between the hints. It is a fixed schedule that rotates between $H_{0}, H_{1}, \cdots, H_{M}$. Thus, at step $k$, a batch of $N$ examples of $H_{m}$ is processed, where $m=k \bmod (M+$ 1). This simple-minded algorithm tends to do well in situations where the $E_{m}$ 's are somewhat similar.

Weighted Rotation: This is the next step in fixed schedules that tries to give different emphasis to different $E_{m}$ 's. The schedule rotates between the hints, visiting $H_{m}$ with frequency $\nu_{m}$. The choice of the $\nu_{m}$ 's can achieve balance by emphasizing the hints that are more important or harder to learn.

Maximum Error: This is the simplest adaptive schedule that tries to achieve the same type of balance as simple rotation. At each step $k$, the algorithm processes the hint with the largest error $E_{m}$. The algorithm uses estimates of the $E_{m}$ 's to make its selection.

Maximum Weighted Error: This is the adaptive counterpart to weighted rotation. It selects the hint with the largest value of $\nu_{m} E_{m}$. The choice of the $\nu_{m}$ 's can achieve balance by making up for disparities between the numerical ranges of the $E_{m}$ 's. Again, the algorithm uses estimates of the $E_{m}$ 's.

Adaptive schedules attempt to answer the question: Given a set of values for the $E_{m}$ 's, which hint is the most under-learned? The above schedules answer the question by comparing the individual $E_{m}$ 's. Although this works well in simple cases, it does not take into consideration the correlation between different hints. As we deal with more and more hints, the correlation between the $E_{m}$ 's becomes more significant. This leads us to the final schedule that achieves the balance between the $E_{m}$ 's through their relation to the actual error $E$.

Adaptive Minimization: Given the estimates of $E_{0}, E_{1}, \cdots, E_{M}$, make $M+1$ estimates of $E$, each based on all but one of the hints:

$$
\begin{gathered}
\hat{E}\left(\bullet, E_{1}, E_{2}, \cdots, E_{M}\right) \\
\hat{E}\left(E_{0}, \bullet, E_{2}, \cdots, E_{M}\right) \\
\hat{E}\left(E_{0}, E_{1}, \bullet, \cdots, E_{M}\right) \\
\cdots \\
\hat{E}\left(E_{0}, E_{1}, E_{2}, \cdots, \bullet\right)
\end{gathered}
$$

and choose the hint for which the corresponding estimate is the smallest.

In other words, $E$ becomes the common thread between the $E_{m}$ 's. Knowing that we are really trying to minimize $E$, and that the $E_{m}$ 's are merely a vehicle to this end, the criterion for balancing the $E_{m}$ 's should be based on what is happening to $E$ as far as we can tell.

\section{References}

Abu-Mostafa, Y. S. (1990), Learning from hints in neural networks, Journal of Complexity 6, 192198.

Abu-Mostafa. Y. S: (1993), A method for learning from hints, Advances in Neural Information Processing Systems - 5, S. Hanson et al (eds), Morgan Kaufmann, 1993.

Al-Mashouq, K. and Reed, I. (1991), Including hints in training neural networks, Neural Computation 3, 418-427.

Minsky, M. L. and Papert, S. A. (1969), "Perceptrons," MIT Press.

Omlin, C. and Giles, C. L. (1992), Training second-order recurrent neural networks using hints, Machine Learning: Proceedings of the Ninth International Conference (ML-92), D. Sleeman and P. Edwards (ed.), Morgan Kaufmann.

Suddarth, S. and Holden, A. (1991), Symbolic neural systems and the use of hints for developing complex systems, International Journal of Machine Studies 35, p. 291.

Towell, G. and Shavlik, J. W. (1992), Interpretation of artificial neural networks: mapping knowledge-based neural networks into rules; $A d$ vances in Neural Information Processing Systems - 4, J. Moody et al (eds), Morgan Kaufmann, 1992.

Wismer, D. A. and Chattergy, R. (1978), "Introduction to Nonlinear Optimization," North Holland.

This work was supported by the United States AFOSR under Grant No. F49620-92J-0398. 